\title{
Analysis and Calculation of Rotor Currents for Doubly Fed Induction Generator at An Early Stage of Terminal Voltage Dip
}

\author{
Wang Jian ${ }^{1,2, a}$, Yan Gangui ${ }^{2}$, Sun Yong ${ }^{3}$, Zheng Chao ${ }^{4}$, Shen Qingkun ${ }^{2}$ \\ ${ }^{1}$ School of Electrical and Electronic Engineering, North China Electric Power University, Beijing, \\ China \\ ${ }^{2}$ School of Electrical Engineering, Northeast Dianli University, Jilin, Jilin Province, China \\ ${ }^{3}$ Dispatching center of Jilin Electric Power Corporation Limited, Changchun, Jilin Province, China \\ ${ }^{4}$ China Electric Power Research Institute, Beijing,100192, China \\ awangjian0122000@126.com
}

Keywords: doubly fed induction generators(DFIG). voltage dip. Crowbar protection. rotor current

\begin{abstract}
It is important to analyze rotor current quantitatively for doubly fed induction generator(DFIG) initiated by terminal voltage dip for planning and configuration of relay protection of wind farms and grid including wind farms. The previous studies assumpt that the instantaneous activation of crowbar after the occurrence of terminal voltage dip. In fact, crowbar acts with different time delays depending on the severity of terminal voltage dip. Based on superposition principle, the terminal voltage is divided into steady-state value and increment. On condition that excitation voltage of rotor windings and rotating speed of rotor keep unchanged, the analytic model to calculate the increment of rotor current initiated by the increment of terminal voltage is derived in detail when crowbar is not triggered. Calculated results of the analytic model is compared with the numerical calculation, which verifies the effectiveness of the proposed analytic model.
\end{abstract}

\section{Introduction}

Currently, a wind turbine with a doubly fed induction generator (DFIG) is one of the mainstream types used by wind power. A power electronic converter which can be divided into the rotor side converter and grid side converter according to the location is used for excitation on the rotor winding.

In order to avoid breakdown of the rotor side converter switches, a crowbar is connected to the rotor windings. When the rotor currents reaches the setting value of protection, the rotor side converter is blocked and the crowbar is fired. Then the rotor windings will be short circuited.

At present, many studies have been proposed that analyze the rotor current transient characteristics when the terminal voltage of DFIGs drops [1, 2, 3]. Most of the previous studies assume that the crowbar is fired at the moment of the appearance of voltage dip. But in fact, time is needed for the rotor current rising up to trigger crowbar [4]. Therefore, the previous studies can not reflect the transient characteristics of the rotor currents from the occurrence of the voltage dips to the moment of crowbar fired(called an early stage of the terminal voltage dip).

Aiming at the question, the terminal voltage of the DFIG is divided into steady-state value and increment, and then based on the superposition principle, the transient characteristics of the rotor current at an early stage of the terminal voltage dip is equivalent to the increment analysis of the rotor currents. Taking the amplitude and the initial phase of the rotor windings excitation voltage and the rotor speed unchanged as the research condition, the analysis calculation model between the terminal voltage increment and the rotor currents increment is derived in detail at an early stage of the terminal voltage dip in a dq synchronously rotating reference frame. The results of case studies carried out with a Gamesa G58-850kW wind turbine with DFIG verify the validity of the proposed method.

\section{Electromagnetic transient model of DFIGs}

In a $d q$ synchronously rotating reference frame, the equations describing a DFIG are $[5,6]$ 


$$
\dot{X}=A(X) X+B U
$$

Where, $X=\left[-i_{\mathrm{s} d},-i_{\mathrm{s} q}, i_{\mathrm{r} d}, i_{\mathrm{r} q}\right]^{\prime}, U=\left[u_{\mathrm{s} d}, u_{\mathrm{s} q}, u_{\mathrm{r} d}, u_{\mathrm{r} q}\right]^{\prime}, u_{\mathrm{s} d}, u_{\mathrm{s} q}, u_{\mathrm{r} d}$ and $u_{\mathrm{r} q}, i_{\mathrm{s} d}, i_{\mathrm{s} q}, i_{\mathrm{r} d}$ and $i_{\mathrm{r} q}$ are stator and rotor $d$ and $q$ axis voltage and current components respectively,

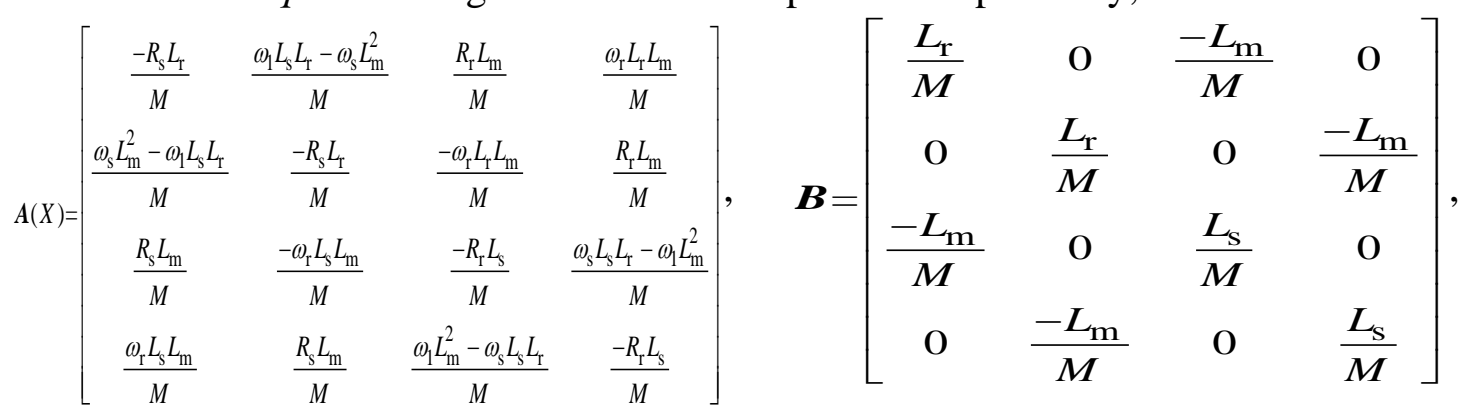

$\omega_{\mathrm{r}}$ is the rotor angular speed, $L_{\mathrm{s}}=L_{\mathrm{sl}}+1.5 L_{\mathrm{sm}}, L_{\mathrm{r}}=L_{\mathrm{rl}}+1.5 L_{\mathrm{sm}}\left(N_{\mathrm{r}} / N_{\mathrm{s}}\right)^{2}, L_{\mathrm{m}}=1.5 L_{\mathrm{sm}}\left(N_{\mathrm{r}} / N_{\mathrm{s}}\right), M=L_{\mathrm{s}} L_{\mathrm{r}}$ $-\left(L_{\mathrm{m}}\right)^{2}, R_{\mathrm{s}}, R_{\mathrm{r}}, L_{\mathrm{sl}}$ and $L_{\mathrm{sr}}$ are the stator and rotor resistance and leakage inductance respectively. $L_{\mathrm{sm}}$ is magnetizing inductance. $N_{\mathrm{s}}$ and $N_{\mathrm{r}}$ are the stator and rotor turns respectively. $\omega_{1}$ is the synchronous rotation angular speed and $\omega_{\mathrm{s}}$ is slip angular speed.

The elements in $A(X)$ are related to winding parameters of DFIG and $\omega_{\mathrm{r}}$. While $\omega_{\mathrm{r}}$ is associated with wind speed, the stator and rotor currents (i.e. state variable $X)$. So, the elements in $A(X)$ are related to the stator and rotor currents. Therefore, Eq. (1) is a nonlinear model.

When $\omega_{\mathrm{r}}$ varys in a small range, $A(X)$ can be constant. So, Eq. (1) degenerates to a linear model, and the superposition principle may be applied to analyze the characteristics of the rotor current stimulated by the terminal voltage dip.

In normal state,

$$
\dot{X}_{0}=A\left(X_{0}\right) X_{0}+B U_{0}=0
$$

Where, the subscript "0" represents the corresponding variables before the voltage dip. $U_{0}=\left[u_{\mathrm{s} d 0}\right.$, $\left.u_{\mathrm{s} q 0}, u_{\mathrm{r} d 0}, u_{\mathrm{r} q 0}\right]^{\prime}, X_{0}=\left[-i_{\mathrm{s} d 0},-i_{\mathrm{s} q 0}, i_{\mathrm{r} d 0}, i_{\mathrm{r} q 0}\right]^{\prime}$.

Assuming that $U$ dropped from $U_{0}$ to $U_{1}$, according to the superposition principle, $U_{1}$ can be regarded as an incremental $\Delta U$ superimposed on $U_{0}$, as shown in Eq.(3).

$$
U_{1}=U_{0}+\Delta U
$$

Where, $\boldsymbol{U}_{\mathbf{1}}$ is the voltage vectors of the stator and rotor after the terminal voltage dip. $\Delta U$ is the vectors of the stator and rotor voltage increment, $\Delta U=\left[\Delta u_{\mathrm{s} d}, \Delta u_{\mathrm{s} q}, \Delta u_{\mathrm{r} d}, \Delta u_{\mathrm{rq}}\right]$ '.

According to the superposition principle, $X$ is

$X=X_{0}+\Delta X$

Where, $\Delta X$ is the vectors of the stator and rotor currents increment stimulated by $\Delta U$ along, $\Delta X=\left[-\Delta i_{s \mathrm{~d}},-\Delta i_{\mathrm{sq}}, \Delta i_{r \mathrm{~d}}, \Delta i_{r q}\right]$.'

Substituting Eq. (2), Eq. (3), Eq. (4) into Eq. (1), the quantitative relationship between $\Delta U$ and $\Delta X$ can be derived, as shown in Eq.(5).

$$
\Delta \dot{X}=A\left(X_{0}\right)(\Delta X)+B(\Delta U)
$$

\section{Calculation model of the rotor currents increment stimulated by the terminal voltage increment of DFIGs}

It's difficult to obtain the analytical expression between $\Delta U$ and $\Delta X$ by solving Eq. (5). Additional, Eq.(5) can not reveal the mechanism between $\Delta U$ and $\Delta X$ from the physical processes perspective and can not grasp the characteristics of the rotor currents increment intuitively.

\section{Analysis of frequency components of the rotor currents increment of DFIG}

Logically, the steady-state value of the stator and rotor currents will change at the beginning of the terminal voltage dip. So, in a $d q$ synchronously rotating reference frame, the DC components should be involved in $\Delta X$.

Meanwhile, in order to maintain the flux linkage of the stator and rotor winding invariable at the moment of the voltage dip, the initial value of the flux linkage of the stator and rotor windings will 
excite free components involved in $\Delta X$.

When the amplitude and initial phase of the rotor excitation voltage remains invariable, in a $d q$ synchronously rotating reference frame, the free components involved in $\Delta X$ are as follows:

(1) The initial value of the stator windings flux linkage will stimulate the freedom current $\Delta i_{s d(q)}$ whose oscillation frequency is $\omega_{1}$ in the stator windings, and the freedom current $\Delta i_{\operatorname{srd}(q)}$ whose oscillation frequency is $\omega_{1}$ in the rotor windings.

(2) The initial value of the rotor windings flux linkage will stimulate the freedom current $\Delta i_{\mathrm{rd}(q)}$ whose oscillation frequency is $\omega_{\mathrm{s}}$ in the rotor windings, and the freedom current $\Delta i_{\mathrm{rsd}(q)}$ whose oscillation frequency is $\omega_{\mathrm{s}}$ in the stator windings.

From the above, at an early stage of the terminal voltage dip, when the amplitude and initial phase of the rotor excitation voltage remains invariable, the increment of the rotor currents mainly contains a constant DC component, the freedom current components whose oscillation frequency are $\omega_{1}$ and $\omega_{\mathrm{s}}$ respectively in a $d q$ synchronously rotating reference.

Due to the existence of $R_{\mathrm{s}}$ and $R_{\mathrm{r}}$, as time goes on, all the the freedom current components of the increment of the rotor currents will decay to zero in accordance with the different time constants.

\section{Analysis of the decay time constant of the rotor freedom currents of DFIGs}

Because $A(X)$ is a 4-order non-sparse matrix, it becomes more tedious to calculate the time constant using rigorous mathematical methods. According to [6], in a $d q$ synchronously rotating reference, the freedom current component in the rotor windings whose oscillation frequency is $\omega_{1}$ will decay following the time constant $T_{\mathrm{sd}(q)}$ of the stator windings, and the freedom current component whose oscillation frequency is $\omega_{\mathrm{s}}$ will decay following the time constant $T_{\mathrm{rd}(q)}$ of the rotor windings.

Supposing that the rotor structure of DFIG is symmetrical in $d$ axis and $q$ axis direction, then $T_{\mathrm{s} d}=T_{\mathrm{s} q}=T_{\mathrm{s}}, T_{\mathrm{r} d}=T_{\mathrm{r} q}=T_{\mathrm{r}}$. According to [6], $T_{\mathrm{s}}$ and $T_{\mathrm{r}}$ are

$$
T_{\mathrm{S}}=\left(L_{\sigma \mathrm{S}}+L_{\mathrm{m}} L_{\sigma \mathrm{rr}}\left(\frac{N_{\mathrm{s}}}{N_{\mathrm{r}}}\right)^{2}\right) /\left(R_{\mathrm{S}}\left(L_{\mathrm{m}}+\frac{N_{\mathrm{s}}}{N_{\mathrm{r}}} L_{\mathrm{\sigma r}}\right)\right), T_{\mathrm{r}}=\left(L_{\mathrm{\sigma r}}+L_{\mathrm{m}} L_{\mathrm{\sigma S}}\left(\frac{N_{\mathrm{r}}}{N_{\mathrm{S}}}\right)^{2}\right) /\left(R_{\mathrm{r}}\left(L_{\mathrm{m}}+\frac{N_{\mathrm{r}}}{N_{\mathrm{S}}} L_{\sigma \mathrm{S}}\right)\right)
$$

\section{The calculation model of the rotor currents increment of DFIGs}

Because of assuming $\Delta u_{\mathrm{rd}(q)}=0$, the amplitude of the freedom current component in the rotor windings whose oscillation frequency is $\omega_{\mathrm{s}}$ is relatively small and could be ignored.

Because the value of $R_{\mathrm{s}}$ and $R_{\mathrm{r}}$ are smaller, ignoring them. So, $A(X)$ turns to $A_{1}(X), A_{1}(X)=$

$$
\left[\begin{array}{cccc}
0 & \frac{\omega_{1} L_{\mathrm{s}} L_{\mathrm{r}}-\omega_{\mathrm{s}} L_{\mathrm{m}}^{2}}{M} & 0 & \frac{\omega_{\mathrm{r}} L_{\mathrm{r}} L_{\mathrm{m}}}{M} \\
\frac{\omega_{\mathrm{S}} L_{\mathrm{m}}^{2}-\omega_{1} L_{\mathrm{s}} L_{\mathrm{r}}}{M} & 0 & \frac{-\omega_{\mathrm{r}} L_{\mathrm{r}} L_{\mathrm{m}}}{M} & 0 \\
0 & \frac{-\omega_{\mathrm{r}} L_{\mathrm{s}} L_{\mathrm{m}}}{M} & 0 & \frac{\omega_{\mathrm{s}} L_{\mathrm{s}} L_{\mathrm{r}}-\omega_{1} L_{\mathrm{m}}^{2}}{M} \\
\frac{\omega_{\mathrm{r}} L_{\mathrm{s}} L_{\mathrm{m}}}{M} & 0 & \frac{\omega_{1} L_{\mathrm{m}}^{2}-\omega_{\mathrm{s}} L_{\mathrm{s}} L_{\mathrm{r}}}{M} & 0
\end{array}\right]
$$

After using the Laplace transformation to Eq.(5), we can get Eq.(6).

$$
\left[S \boldsymbol{E}-\boldsymbol{A}_{1}\left(\boldsymbol{X}_{0}\right)\right][\Delta \boldsymbol{X}(S)]=\boldsymbol{B}[\Delta \boldsymbol{U}(S)]
$$

Where, $S$ is complex variable, $E$ is Unit Matrix, $\Delta X(S)$ and $\Delta U(S)$ are the Laplace transformation for $\Delta X$ and $\Delta U$ respectively.

When the terminal voltage drops, $\Delta U$ is constant. So the Laplace transformation of $\Delta i_{\operatorname{rd}(q)}$ is

$$
\left\{\begin{array}{l}
\Delta i_{\mathrm{r} d}(S)=\frac{L_{\mathrm{m}}}{M}\left[\frac{\Delta u_{\mathrm{s} d}}{S^{2}+\omega_{1}^{2}}-\frac{\Delta u_{\mathrm{s} q} \times \omega_{1}}{S\left(S^{2}+\omega_{1}^{2}\right)}\right] \\
\Delta i_{\mathrm{r} q}(S)=\frac{L_{\mathrm{m}}}{M}\left[\frac{\Delta u_{\mathrm{s} q}}{S^{2}+\omega_{1}^{2}}-\frac{\Delta u_{\mathrm{s} d} \times \omega_{1}}{S\left(S^{2}+\omega_{1}^{2}\right)}\right]
\end{array}\right.
$$

Using the inverse Laplace transformation to Eq.(7), then 


$$
\left\{\begin{array}{l}
\Delta i_{\mathrm{i} d}(t)=\frac{L_{\mathrm{m}}}{M \omega_{1}}\left[\Delta u_{\mathrm{sq}}+\Delta u_{\mathrm{sd}} \times \sin \left(\omega_{1} t\right)-\Delta u_{\mathrm{sq}} \times \cos \left(\omega_{1} t\right)\right]=\frac{L_{\mathrm{m}}}{M \omega_{\mathrm{l}}}\left[\Delta u_{\mathrm{sq}}+\sqrt{\left(\Delta u_{\mathrm{s} d}\right)^{2}+\left(\Delta u_{\mathrm{sq}}\right)^{2}} \sin \left(\omega_{1} t+c_{1}\right)\right] \\
\Delta i_{\mathrm{rq}}(t)=\frac{L_{\mathrm{m}}}{M \omega_{1}}\left[-\Delta u_{\mathrm{sd}}+\Delta u_{\mathrm{sq}} \times \sin \left(\omega_{1} t\right)+\Delta u_{\mathrm{sd}} \times \cos \left(\omega_{1} t\right)\right]=\frac{L_{\mathrm{m}}}{M \omega_{1}}\left[-\Delta u_{\mathrm{sd}}+\sqrt{\left(\Delta u_{\mathrm{sd}}\right)^{2}+\left(\Delta u_{\mathrm{sq}}\right)^{2}} \sin \left(\omega_{1} t+c_{2}\right)\right]
\end{array}\right.
$$

Where, $c_{1}=\arctan \left(-\Delta u_{\mathrm{s} q} / \Delta u_{\mathrm{s} d}\right), c_{2}=\arctan \left(\Delta u_{\mathrm{s} d} / \Delta u_{\mathrm{s} q}\right)$.

Modifying Eq.(8) [6], considering the damping effect of $R_{s}$ and $R_{r}, \Delta i_{\mathrm{rd}(q)}$ is

$$
\left\{\begin{array}{l}
\Delta i_{\mathrm{rd}}(t)=\frac{L_{\mathrm{m}}}{M \omega_{1}}\left[\Delta u_{\mathrm{sq}}+\sqrt{\left(\Delta u_{\mathrm{sd}}\right)^{2}+\left(\Delta u_{\mathrm{sq}}\right)^{2}} \sin \left(\omega_{1} t+c_{1}\right) e^{-t T \cdot}\right] \\
\Delta i_{\mathrm{rq}}(t)=\frac{L_{\mathrm{m}}}{M \omega_{1}}\left[-\Delta u_{\mathrm{sd}}+\sqrt{\left(\Delta u_{\mathrm{sd}}\right)^{2}+\left(\Delta u_{\mathrm{sq}}\right)^{2}} \sin \left(\omega_{1} t+c_{2}\right) e^{-t T T_{\mathrm{s}}}\right]
\end{array}\right.
$$

Assuming that the voltage oriented control with the constant amplitude, the stator voltage vector is oriented to $q$ axis, then $u_{\mathrm{s} d \mathbf{0}}=\mathbf{0}, \boldsymbol{u}_{\mathrm{s} q \mathbf{0}}=\sqrt{2 / 3} U_{\mathrm{s}}$. Where, $U_{\mathrm{s}}$ is the effective value of stator line voltage.

Ignoring the phase jump caused by the terminal voltage dip, then $\Delta u_{\mathrm{s} d}=0, \Delta u_{\mathrm{sq}}>0, c_{1}=-\pi / 2, c_{2}=0$.

Eq.(9) changes to Eq.(10).

$$
\left\{\begin{array}{l}
\Delta i_{\mathrm{r} d}(t)=\frac{L_{\mathrm{m}}}{M \omega_{1}}\left[\Delta u_{\mathrm{s} q}-\Delta u_{\mathrm{s} q} \cos \left(\omega_{1} t\right) e^{\left.-t / T_{\mathrm{r}}\right]}\right. \\
\Delta i_{\mathrm{r} q}(t)=\frac{L_{\mathrm{m}}}{M \omega_{1}}\left[\Delta u_{\mathrm{s} q} \sin \left(\omega_{1} t\right) e^{-t / T_{\mathrm{r}}}\right]
\end{array}\right.
$$

According to Eq.(10),

(1) When $t_{\mathrm{rd}}=\left[\arctan \left(T_{\mathrm{s}} \omega_{1}\right)+\arctan \left(\Delta u_{\mathrm{sq}} / \Delta u_{\mathrm{sd}}\right)+k_{1} \pi\right] / \omega_{1}, \Delta i_{\mathrm{rd}}$ reaches a maximum $\Delta i_{\mathrm{rdmax}}$. Where, $k_{1}=0$ when $\left[\arctan \left(T_{\mathrm{s}} \omega_{1}\right)+\arctan \left(\Delta u_{\mathrm{sq}} / \Delta u_{\mathrm{s} d}\right)\right]>0, k_{1}=1$ when $\left[\arctan \left(T_{\mathrm{s}} \omega_{1}\right)+\arctan \left(\Delta u_{\mathrm{sq}} / \Delta u_{\mathrm{s} d}\right)\right]<0$.

(2) When $t_{\mathrm{r} q}=\left[\arctan \left(T_{\mathrm{s}} \omega_{1}\right)+\arctan \left(-\Delta u_{\mathrm{s} d} / \Delta u_{\mathrm{s} q}\right)+k_{2} \pi\right] / \omega_{1}, \Delta i_{\mathrm{rq}}$ reaches a maximum $\Delta i_{\mathrm{rqmax}}$. Where, $k_{2}=0$ when $\left[\arctan \left(T_{\mathrm{s}} \omega_{1}\right)+\arctan \left(-\Delta u_{\mathrm{s} d} / \Delta u_{\mathrm{s} q}\right)\right]>0, k_{2}=1$ when $\left[\arctan \left(T_{\mathrm{s}} \omega_{1}\right)+\arctan (-\right.$ $\left.\left.\Delta u_{\mathrm{sd}} / \Delta u_{\mathrm{sq}}\right)\right]<0$.

Substituting $t_{\mathrm{rd}}$ and $t_{\mathrm{rq}}$ into Eq. (10), $\Delta i_{\mathrm{rdmax}}$ and $\Delta i_{\mathrm{rqmax}}$ can be derived.

\section{Case Studies}

This section will present case studies carried out with a Gamesa G58-850kW DFIG WTG. A voltage dip is applied to the terminals of the DFIG and the rotor current is calculated by the proposed method. The results are compared with those calculated by Eq.(1) through numerical calculations to verify the validity of the proposed method. The parameters of the WTG are listed in Table 1.

Table 1 Coefficients of a Gamesa G58-850kW DFIG

\begin{tabular}{cccccc}
\hline Rated power/kW & Rotor speed/(r· $\left.\min ^{-1}\right)$ & Stator voltage/V & Rotor voltage/V Rotor current/A & $N_{\mathrm{s}}$ \\
\hline \hline 850 & 1616 & 690 & 150 & 277 & 690 \\
\hline$N_{\mathrm{r}}$ & $R_{\mathrm{s}} / \Omega$ & $X_{\mathrm{s}} / \Omega$ & $R_{\mathrm{r}} / \Omega$ & $X_{\mathrm{r}} / \Omega$ & $X_{\mathrm{m}} / \Omega$ \\
\hline 1852 & 0.016 & 0.074 & 0.0125 & 0.121 & 6.78 \\
\hline
\end{tabular}

Where, the windings of stator is connected in $\Delta$ and the windings of rotor is connected in $\mathrm{Y}$ and parameters of the rotor side are not reduced to the stator side.

Taking the rated operating condition as test conditions, it is assumed that the crowbar is not connected to the during the test.

When $t=0 \mathrm{~s}$, the terminal voltage drops to 0.9p.u. , and $\Delta u_{\mathrm{s} d}=0, \Delta u_{\mathrm{s} q}=0.1 \times \sqrt{2 / 3} U_{\mathrm{s}}=56.34(V)$.

According to Eq.(1), and Eq.(10), the results of $\Delta i_{\mathrm{rd}(q)}$ calculated by Eq.(1) through numerical calculations and the proposed method respectively is shown in Fig.1.
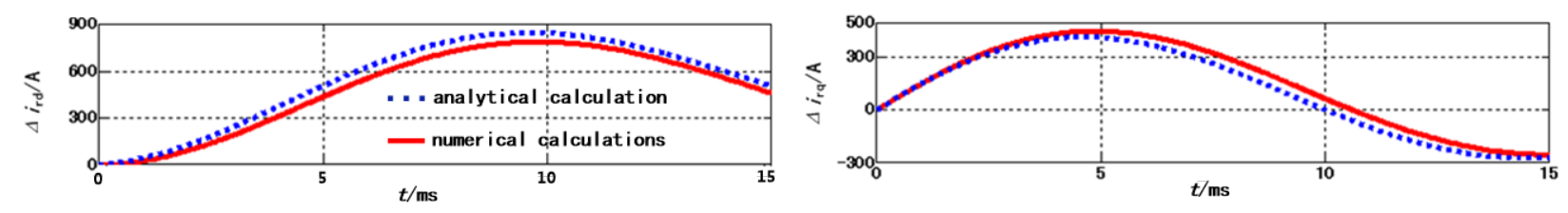

Fig.1 Curve of $\Delta i_{\mathrm{rd}}$ and $\Delta i_{\mathrm{rq}}$ 
It is shown in Fig.4 that the results of $\Delta i_{\text {rd(q) }}$ calculated by the proposed method and Eq.(1) through numerical calculations agree well. The maximums calculated by the two methods are close, as shown in Table 2.

Table 2 Calculating results of $\Delta i_{\mathrm{rdmax}}, \Delta i_{\mathrm{r} q \max }, t_{\mathrm{r} d}$ and $t_{\mathrm{r} q}$ at the rated operating condition

\begin{tabular}{cccc}
\hline variable & By numerical calculations/A & By the proposed method/A & relative error $/ \%$ \\
\hline$\Delta i_{\text {rdmax }}$ & 869.43 & 844.51 & 2.87 \\
$\Delta i_{\text {rqmax }}$ & 455.57 & 415.78 & 8.81 \\
\hline \hline variable & By numerical calculations $/ \mathrm{ms}$ & By the proposed method $/ \mathrm{ms}$ & relative error $/ \%$ \\
\hline$t_{\mathrm{r} d}$ & 10 & 9.6 & 4 \\
$t_{\mathrm{r} q}$ & 5 & 4.6 & 8 \\
\hline
\end{tabular}

\section{Conclusions}

On condition that excitation voltage of rotor windings and rotating speed of rotor keep unchanged, the electromagnetic transient process at an early stage of terminal voltage dip are analyzed when Crowbar protection is not triggered. Neglecting the stator and rotor resistance, the analytic model to calculate the increment of rotor current initiated by the increment of terminal voltage is derived in detail. Calculated results of the analytic model is compared with the numerical calculation, which verifies the effectiveness of the proposed analytic model.

The physical meaning of the proposed method is clear. The proposed method is convenient for engineering applications and is contributed to the quantitative analysis of electromagnetic dynamics of DFIGs stimulated by voltage dips.

\section{Acknowledgements}

The work is supported by the National Basic Research Program of China(973 Program) (Grant No. 2013CB228201).

\section{References}

[1] Petersson A, Thiringer T, Harnefors L, et al. Modeling and experimental verification of grid interaction of a DFIG wind turbine[J]. IEEE Transactions on Energy Conversion, 2005, 20(4): 878-885.

[2] M.Z. Cheng, Z.L. Dou, J.W. Zhang, et al. Transient analysis of DFIG with a crowbar circuit during the voltage dip[J]. Power System and Clean Energy, 2012, 28(5): 54-60.

[3] Lopez J, Sanchis P, Roboa X. Dynamic behavior of the doubly fed induction generator during three-phase voltage dips[J]. IEEE Transactions on Energy Conversion, 2007, 22(3): 709-717.

[4] Petersson A. Analysis, modeling and control of doubly-fed induction generators for wind turbines[D]. Goteborg, Sweden: Chalmers University of Technology, 2005.

[5] Lopez J, Gubia E, Sanchis P, et al. Wind turbines based on doubly fed induction generator under asymmetrical voltage[J]. IEEE Transactions on Energy Conversion, 2008, 23(1): 321-330.

[6] Morren J, Haan S W H. Short-circuit current of wind turbines with doubly fed induction generator[J]. IEEE Transactions on Energy Conversion, 2007, 22(1): 174-180. 